\title{
Acid sphingomyelinase, autophagy, and atherosclerosis
}

\author{
Friedrich C. Luft
}

Published online: 2 March 2014

(C) Springer-Verlag Berlin Heidelberg 2014

Sphingolipids are ubiquitous membrane lipids responsible for a daunting array of cellular functions and are critical to a host of metabolic diseases. Sphingolipid products, such as sphingosine-1-phosphate, operate as tissue hormones and signaling molecules with their own receptors and are already being targeted in human diseases [1]. A series of enzymes are important to sphingolipid metabolism. Sphingomyelinases are enzymes that catalyze the cleavage of the phosphodiester bond from sphingomyelin. The cleavage results in the production of ceramide and phosphocholine (Fig. 1). Five sphingomyelinases are known and classified as acid, basic, or neutral sphingomyelinases. The lysosomal acidic sphingomyelinase (ASM) is involved in the production of ceramide in the cellular response to environmental insults, infection with pathogens, ligation of death receptors, drugs, and other stressors. The main mechanism of trafficking acid sphingomyelinase to the lysosome is through the mannose 6phosphate receptor mediated pathway. Clinicians will recall that ASM deficiency brought about by mutations in the encoding gene, SMPD1, is the cause of Niemann-Pick disease type A and type B. As a result, toxic amounts of sphingomyelin are present in all cells throughout the body [2].

Atherosclerosis occurs from the thickening of artery walls through depositing of cholesterol and triglyceride largely in vascular smooth muscle cells (VSMC). High levels of circulating low-density lipoprotein cholesterol (LDL) often caused by inadequate removal by high-densitiy lipoprotein particles (HDL) particles are associated with atherosclerosis. ASM plays a role in atherosclerosis by promoting aggregation of lipoproteins to arterial walls. ASM is suggested to be one of the key enzymes responsible for low-density lipoprotein

F. C. Luft $(\bowtie)$

Experimental and Clinical Research Center, Charité Medical Faculty,

Berlin, Germany

e-mail: luft@charite.de cholesterol aggregation during atherogenesis. Indeed, in aggregated LDL isolated from human atherosclerotic lesions, the ceramide content is 10-50-fold higher than in corresponding plasma LDL. Furthermore, treatment of LDL with human recombinant secretory ASM in vitro induces the formation of lesion-like LDL aggregates. Sneck et al. [3] showed that sphingomyelin hydrolysis induces exposure of proteasesensitive sites of apoB-100, whose interactions govern subsequent particle aggregation. They found that supersized LDL aggregates could contribute to the retention of LDL lipids in acidic areas of atherosclerosis-susceptible sites in the arterial intima. ASM inhibition has been considered as a therapeutic target for treating atherosclerosis.

In this issue of $J \mathrm{Mol} \mathrm{Med}$, Li et al. report on how ASM influences autophagy in vascular smooth muscle cells (VSMC) from coronary arteries [4]. They relied on Smpd1 gene-deficient mice and Smpdl-intact control mice; the mice were given a Western diet. The VSMC from these mice expressed robust amounts of microtubule-associated protein1 light chain-3 (LC3B). LC3B is an ubiquitin-like protein that is a constituent of the ATG8-conjugation system, one of two evolutionarily conserved phosphatidylethanolamine conjugation systems necessary for the formation of the autophagosome (AP), as well as a protein called p62. The p62 protein recognizes toxic cellular waste, which is then scavenged by a sequestration process known as self-eating, namely autophagy [5]. 7-Ketocholesterol is a major oxidation product of cholesterol found in human atherosclerotic plaques and is more atherogenic than cholesterol. 7-Ketocholesterol also induces autophagy. Li et al. treated VSMC from intact mice with 7-ketocholesterol and observed upregulation of LC3B and increased formation of APs as well as autophagolysosomes (APLs). In cells from the Smpd1 gene-deleted mice, the LC3B expression and APs were increased still further, compared to intact cells. However, the formation of APLs was markedly diminished. The authors next relied on fluorescence resonance 


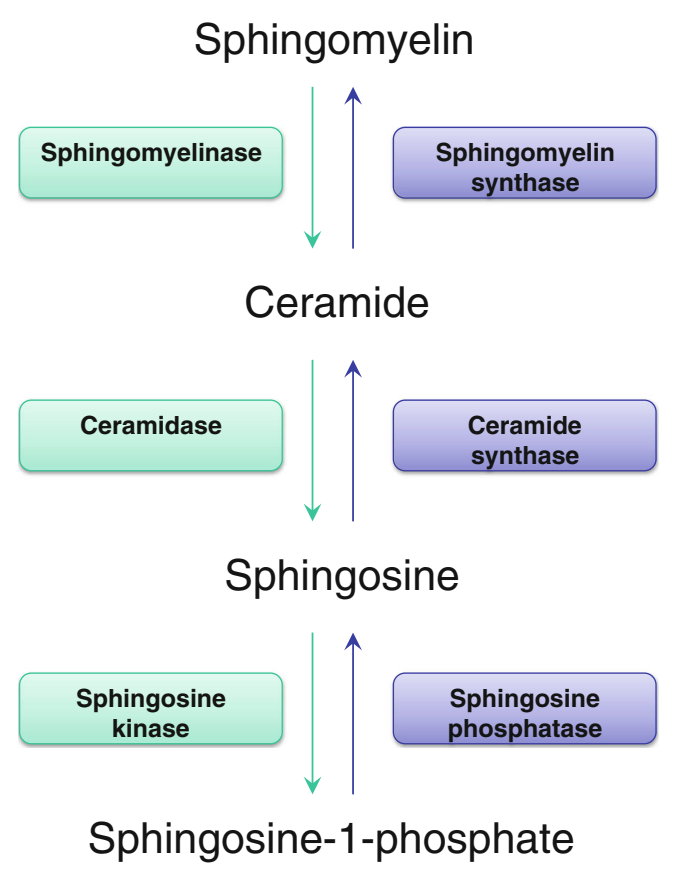

Fig. 1 Sphingomyelin is converted to ceramide by acid sphingomyelinase. Ceramide is transported from the endoplasmic reticulum to the Golgi. Ceramide can be metabolized to sphingosine, which in turn can be phosphorylated to sphingosine-1-phosphate

energy transfer (FRET) between fluorescence-labeled LC3B and the lysosomal marker, Lamp1. They found that 7-ketocholesterol markedly induced fusion of APs with lysosomes in Smpdl-intact derived VSMC. However, this fusion did not occur in cells derived from Smpdl gene-deleted mice. The authors also observed that cell proliferation was enhanced in VSMC from Smpdl gene-deleted mice, and indeed the cell expression of the dedifferentiation marker, vimentin, was increased. When the authors then overexpressed ASM into the Smpdl genedeleted cells, the formation of APLs was restored. The authors interpret their data as indicating an important role for ASM in controlling lysosomal trafficking and fusion to enable normal autophagy. Malfunction of VSMC autophagy could be an important atherogenic mechanism in coronary arteries.

Do we have robust data suggesting that autophagy is important to atherosclerosis? A recent review strongly suggests that this is indeed the case [6]. Most likely, autophagy safeguards plaque cells against cellular distress, in particular, oxidative injury, by degrading the damaged intracellular material. In this way, autophagy would be protective and could contribute to cellular recovery in an unfavorable environment. Pharmacological approaches have recently been developed to stabilize vulnerable, rupture-prone lesions through induction of autophagy. The autophagic process involves formation of doublemembrane structures termed phagophores (Fig. 2). The phagophore expands and, on completion, forms an AP, which then fuses with lysosomes, thereby generating an APL. Incorporation of the outer AP membrane in the lysosomal membrane allows the degradation of the remaining inner single membrane

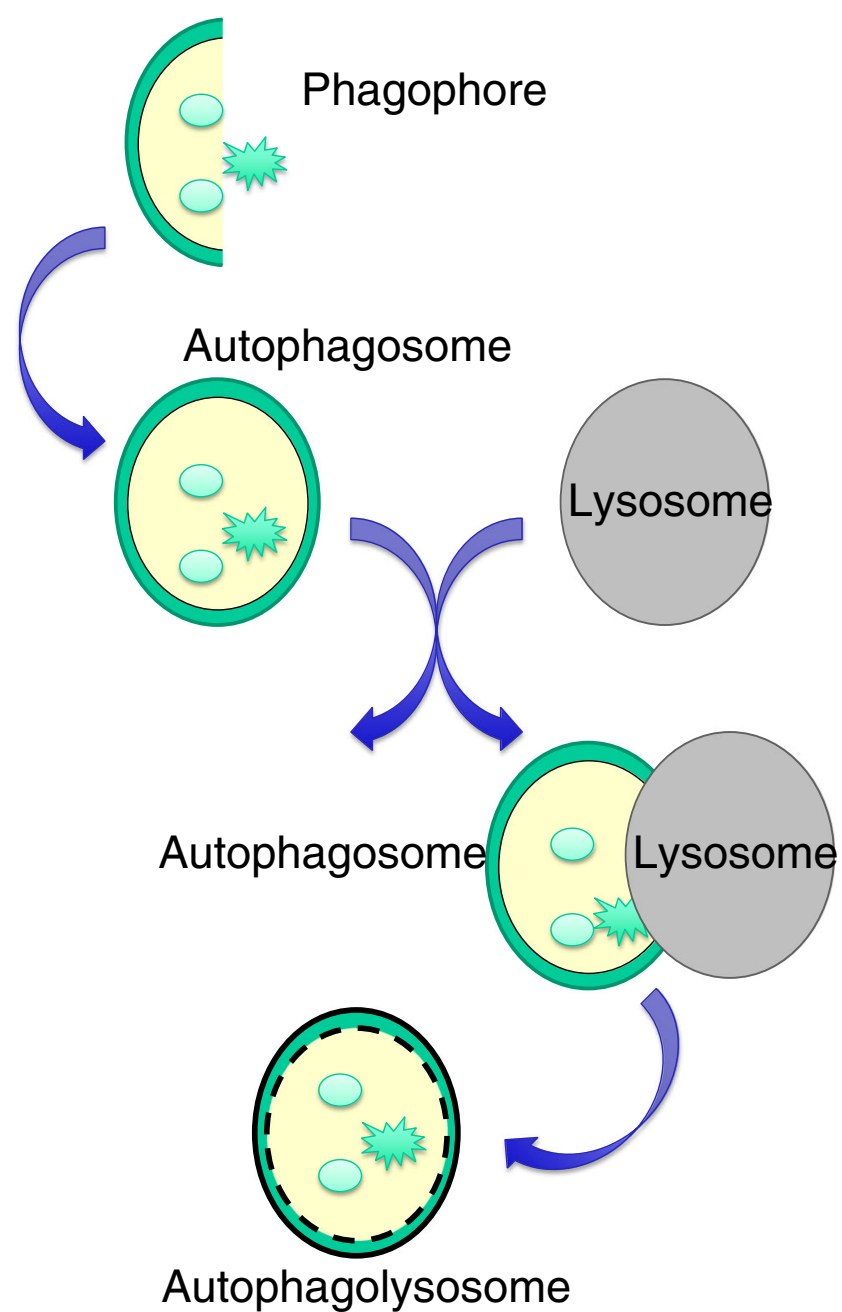

Fig. 2 A phagophore is formed at the initiation of autophagy to sequester cytoplasmic components for degradation. On completion, the phagophore forms a double-membraned vesicle called an autophagosome. After fusion with a lysosome, the content is degraded by lysosomal hydrolases. $\mathrm{Li}$ et al. [4] find that acid sphingomyelinase is necessary for the autophagosome-lysosomal fusion

and the cytoplasmic contents by lysosomal hydrolases. This scheme fits well into what we learned from Li et al. [4].

How could autophagy protect us from atherosclerosis? Basal autophagy protects VSMC from developing into plaque cells by acting against oxidative stress and by degrading damaged intracellular material, particularly polarized mitochondria. Martinet et al. demonstrated the protective role of autophagy in atherosclerosis, at least in vitro [7]. They induced autophagy in VSMCs by giving 7-ketocholesterol. The induction protected the VSMC from cell death as induced by low concentrations of statins. Admittedly, these experiments were a bit counterintuitive. However, $\mathrm{Li}$ et al. also found that 7ketocholesterol induced autophagy [4]. Probably, the engulfment of defective mitochondria by APs limits the release of proapoptotic proteins, such as cytochrome $\mathrm{C}$, into the cytosol.

Do we have evidence from other sources that ASM regulates autophagy? Taniguchi et al. recently reported that 
depleting cells of amino acids caused autophagy with an increase in ASM activity and resultant ceramide production, which serves as an autophagy-inducing lipid [8]. When Taniguchi et al. knocked down ASM in their cells, they successfully decreased autophagy induction. Sphingosine-1phosphate, a product of ceramide, counteracted autophagy induction by amino acid depletion. The authors attributed these effects to regulation by sphingosine-1-phosphate in the mammalian target of rapamycin (mTOR) pathway.

More work is needed is an overused and droll comment. However, the complexity of sphingolipid signaling makes in vivo studies mandatory to support cell-based experiments. The Smpd1 gene-deleted mice will not help us because they develop Niemann-Pick disease [9]. Cell-specific models are needed, perhaps with a conditional knockdown. Studies directed at the highly complex autophagy pathways are also necessary. What is missing is how ASM function influences AP-lysosomal fusion to produce APLs. The authors mention several interesting possibilities including faulty regulation of $\mathrm{Ca}^{2+}$-dependent membrane trafficking or even inhibition or loss of dynein function. Implicating this motor protein is an interesting and bold idea. We can only hope that investigators pick up on it.

Respectfully, Friedrich C. Luft

\section{References}

1. Hia T, Dannenberg AJ (2012) Sphingolipid signaling in metabolic disorders. Cell Metab 16:420-434

2. Henry B, Ziobro R, Becker KA, Kolesnick R, Gulbins E (2013) Acid sphingomyelinase. Handb Exp Pharmacol 215:77-88

3. Sneck M, Nguyen SD, Pihlajamaa T, Yohannes G, Riekkola ML, Milne R, Kovanen PT, Oörni K (2012) Conformational changes of apoB-100 in SMase-modified LDL mediate formation of large aggregates at acidic pH. J Lipid Res 53:1832-1839

4. Li X, Xu M, Pitzer AL, Xia M, Boini KM, Li P-L, Zhang Y (2014) Control of autophagy maturation by acid sphingomyelinase in mouse coronary arterial smooth muscle cells: protective role in atherosclerosis. J Mol Med doi:10.1007/s00109-014-1120-y

5. Rusten TE, Stenmark H (2010) p62, an autophagy hero or culprit? Nat Cell Biol 12:207-209

6. Schrijvers DM, DeMeyer GRY, Martinet W (2011) Autophagy in atherosclerosis. Arterioscler Thromb Vasc Biol 28:1421-1428

7. Martinet W, Schrijvers DM, Timmermans JP, Bult H (2008) Interactions between cell death induced by statins and 7ketocholesterol in rabbit aorta smooth muscle cells. Br J Pharmacol 154:1236-1246

8. Taniguchi M, Kitatani K, Kondo T, Hashimoto-Nishimura M, Asano S, Hayashi A, Mitsutake S, Igarashi Y, Umehara H, Takeya H et al (2012) Regulation of autophagy and its associated cell death by "sphingolipid rheostat": reciprocal role of ceramide and sphingosine 1-phosphate in the mammalian target of rapamycin pathway. J Biol Chem 287:39898-39910

9. Horinouchi K, Erlich S, Perl DP, Ferlinz K, Bisgaier CL, Sandhoff K, Desnick RJ, Stewart CL, Schuchman EH (1995) Acid sphingomyelinase deficient mice: a model of types A and B Niemann-Pick disease. Nat Genet 10(3):288-293 